\section{Decentralized Control and Periodic Feedback}

Pramod P. Khargonekar and A. Bülent Özgüler

\begin{abstract}
The decentralized stabilization problem for linear, discretetime, periodically time-varying plants using periodic controllers is considered. The main tool used is the technique of lifing a periodic system to a time-invariant one via extensions of the input and output spaces. It is shown that a periodically time-varying system of fundamental period $N$ can be stabilized by a decentralized periodic controller if and only if: 1) the system is stabilizable and detectable, and 2) the $\boldsymbol{N}$-lifting of each complementary subsystem of identically zero input-output map is free of unstable input-output decoupling zeros. In the special case of $N=1$, this yields and clarifies all the major existing results on decentralized stabilization of time-invariant plants by periodically timevarying controllers.
\end{abstract}

\section{INTRODUCTION}

This paper is concerned with the time-varying decentralized stabilization problem (TVDSP) in the special case when the plant and the decentralized controller are both periodic. If the plant is time invariant, i.e., periodic with fundamental period equal to 1 , then the decentralized stabilization problem using a periodic controller has the following very significant but partial solution due to Anderson and Moore [2]: If the plant is time invariant, canonical, and strongly connected (see [3]), then there exists a periodic decentralized controller solving TVDSP. This result of [2] (also see the references therein) applies to discrete- as well as continuous-time systems, and their construction is analogous to that of Corfmat and Morse [3] which is concerned with the time-invariant decentralized stabilization problem (TIDSP). They show that with suitable memoryless periodic local controllers applied at $\nu-1$ of the $\nu$ channels of the plant, a reachable and observable time-varying closed-loop system is obtained. This single-channel system can now be uniformly asymptotically stabilized by a dynamic periodically time-varying controller. Wang [23] independently observed the fixed mode elimination property of time-varying controllers, and Juan and Kabamba [9] have obtained related results using generalized hold functions.

In this paper, we use a different approach and utilize the technique of "lifting" to solve the decentralized stabilization problem. Thus, the main contribution of the paper lies in developing new tools for the synthesis of periodic controllers for decentralized stabilization. With these techniques, in Theorem 2 we obtain a more precise form of the above result of [2]. From a technical standpoint, the main improvement is in clarifying the issue as to the necessity of the strong connectedness assumption. There has been some confusion in the literature as to what type of fixed modes can actually be eliminated by periodic feedback; see, e.g., [19]. In Willems [25], this has been clarified to a certain extent, and it has been argued to the effect that: "Only (structurally) fuxed modes. . .caused by the fact

Manuscript received May 26, 1992; revised February 15, 1993. This work was supported in part by the National Science Foundation under Grants INT9101276, ECS-9001371, the Air Force Office of Scientific Research under Contract AFOSR-90-0053, the Army Research Office under Grant DAAL0390-G-0008, and the Scientific and Technical Research Council of Turkey (TÜBITAK) under Grant TBAG-1016.

P. P. Khargonekar is with the Department of Electrical Engineering and Computer Science, University of Michigan, Ann Arbor, MI 48109.

A. B. Özgüler is with the Department of Electrical and Electronics Engineering, Bilkent University, Bilkent, Ankara, 06533 Turkey.

IEEE Log Number 9216452. that the system is not strongly connected are also fixed with respect to time-varying output feedback." Theorem 2 yields a precise statement and a different proof of this fact. An additional advantage of our alternative approach is that it easily yields an extension of this result in Theorem 3 to the case of periodic plants. These results are obtained via the central Theorem 1, which clarifies the relationship of periodic feedback to the elimination of "incompleting zeros" or decentralized fixed modes. The main results obtained here have already found interesting applications in the decentralized simultaneous and reliable stabilization problems [21].

The idea of treating problems involving periodic plants through the transformation of "lifting" originates in Jury and Mullin [10], Sz.-Nagy and Foias [18], Davis [4], and Meyer and Burrus [14], and has been extensively studied by Khargonekar et al. [12] in many feedback control problems of recent interest. The relevance of the techniques developed in [12] to decentralized control has been noted by Davison and Chang [5].

We now define the problems that will be considered.

Let a linear, time-varying, discrete-time, causal system $\Sigma(k)$ of $\nu$-channels be represented by the equations

$$
\begin{aligned}
& \Sigma(k): \quad x(k+1)=A(k) x(k)+\sum_{j=1}^{\nu} B_{j}(k) u_{j}(k), \\
& y_{i}(k)=C_{i}(k) x(k)+\sum_{j=1}^{\nu} D_{i j}(k) u_{j}(k) \text {, } \\
& i=1, \cdots, \nu
\end{aligned}
$$

where for each integer $k \geq 0$, the matrices $A(k), B_{j}(k), C_{j}(k)$, and $D_{i j}(k)$ are real matrices of sizes $n \times n, n \times m_{i}, p_{j} \times n$, and $p_{i} \times m_{j}$, respectively. At each channel $i$, we apply a local controller of the type

$$
\begin{aligned}
\bar{\Sigma}_{i}(k): & \bar{x}_{i}(k+1)=\bar{A}_{i}(k) \bar{x}_{i}(k)+\bar{B}_{i}(k) y_{i}(k) \\
u_{i}(k) & =\bar{C}_{i}(k) \bar{x}_{i}(k)+\bar{D}_{i}(k) y_{i}(k), \\
&
\end{aligned}
$$

where for each integer $k \geq 0$ and for $i=1, \cdots, \nu$, the matrices $\bar{A}_{i}(k), \bar{B}_{i}(k), \bar{C}_{i}(k)$, and $\bar{D}_{i}(k)$ are real matrices of sizes $\bar{n}_{i} \times \bar{n}_{i}$, $\bar{n}_{i} \times p_{i}, m_{i} \times \bar{n}_{i}$, and $m_{i} \times p_{i}$. Under the condition

$$
I-D(k) \bar{D}(k) \text { is nonsingular } \quad \forall k \geq 0,
$$

the resulting autonomous closed-loop system is well defined and is given by the difference equation

$$
\begin{gathered}
{\left[\begin{array}{l}
x(k+1) \\
\bar{x}(k+1)
\end{array}\right]=\left[\begin{array}{cc}
A+B \bar{D}(I-D \bar{D})^{-1} & B(I-\bar{D} D)^{-1} \bar{C} \\
\bar{B}(I-D \bar{D})^{-1} C & \bar{A}+\bar{B} D(I-\bar{D} D)^{-1} \bar{C}
\end{array}\right]} \\
\cdot\left[\begin{array}{l}
x(k) \\
\bar{x}(k)
\end{array}\right]
\end{gathered}
$$

where dependence on $k$ of the matrices $A, \bar{A}$, etc., is not displayed for simplicity, and where $\bar{x}(k):=\left[\bar{x}_{1}(k)^{\prime} \cdots \bar{x}_{\nu}(k)^{\prime}\right]^{\prime}$, denoting the transpose by prime, $\bar{A}(k):=b \operatorname{diag}\left\{\bar{A}_{1}(k), \cdots, \bar{A}_{\nu}(k)\right\}, \bar{B}(k):=$ $b \operatorname{diag}\left\{\bar{B}_{1}(k), \cdots, \bar{B}_{\nu}(k)\right\}, \bar{C}(k):=b \operatorname{diag}\left\{\bar{C}_{1}(k), \cdots, \bar{C}_{\nu}(k)\right\}$, $\bar{D}(k):=b \operatorname{diag}\left\{\bar{D}_{1}(k), \cdots, \bar{D}_{\nu}(k)\right\}$, and

$$
\begin{aligned}
& B(k):=\left[\begin{array}{lll}
B_{1}(k) & \cdots & B_{\nu}(k)
\end{array}\right], C(k):=\left[\begin{array}{c}
C_{1}(k) \\
\vdots \\
C_{\nu}(k)
\end{array}\right] \\
& D(k):=\left[\begin{array}{ccc}
D_{11}(k) & \cdots & D_{1 \nu}(k) \\
\vdots & & \vdots \\
D_{\nu 1}(k) & \cdots & D_{\nu \nu}(k)
\end{array}\right]
\end{aligned}
$$

The resulting controller for (1) is the block-diagonal system $\bar{\Sigma}(k)=(\bar{A}(k), \bar{B}(k), \bar{C}(k), \bar{D}(k))$, and is referred to as a decentralized controller for (1). 
We define the time-varying decentralized stabilization problem (TVDSP) as determining a controller of the form (2) that satisfies (3) such that the autonomous system (4) is uniformly asymptotically stable (u.a.s.). This problem is a generalization to discrete-time, time-varying systems of the well-known decentralized stabilization problem in which both the system (1) and the controller (2) are time invariant [24]. Thus, the time-invariant decentralized stabilization problem (TIDSP) is to determine local controllers of the form (2) for the system (1) (where all the matrices $A, B, C, D, \bar{A}, \bar{B}, \bar{C}, \bar{D}$, are independent of $k$, i.e., constant matrices) such that (3) is satisfied and all the eigenvalues of the state matrix in (4) are inside the open unit disk $\boldsymbol{D}$ of the complex plane.

\section{TI CONTROLleRs for TI Plants}

In this section, we will describe the solvability condition for TIDSP-absence of the unstable decentralized fixed modes of the plant (1) under the depicted structure for the decentralized controller (see [24] and [5]).

Let $\Sigma=(A, B, C, D)$ be a linear, time-invariant, causal, discretetime system with $A \in \boldsymbol{R}^{n \times n}, B \in \boldsymbol{R}^{n \times m}, C \in \boldsymbol{R}^{p \times n}, D \in \boldsymbol{R}^{p \times m}$. The system $\Sigma$ is complete if for all $z \in C$,

$$
\operatorname{rank}\left[\begin{array}{cc}
z I-A & B \\
-C & D
\end{array}\right] \geq n
$$

If the rank condition (5) holds for all $z \in \bar{D}$, where $\bar{D}$ is the complement of the open unit disk, then the system $\Sigma$ is said to be weakly complete. Note that in the definitions above, it is enough to check the rank condition for only the spectrum of $A$ since, for any $z$ which is not an eigenvalue of $A$, the condition is automatically satisfied. Any eigenvalue $z$ of $A$ for which (5) fails will be called an incompleting zero of $\Sigma$. The definition of completeness here is different from the original one given by Corfmat and Morse [3] in that here the condition $C(z I-A)^{-1} B+D \neq 0$ is not imposed. In fact, if the transfer matrix is identically zero, then the system is complete just in case it has no i.o.d. input-output decoupling (i.o.d.) zeros, as we show below. The definitions of zeros used in this paper are quite standard. See the book by Rosenbrock [16] for details. The following result will be useful in establishing our main results.

Lemma 1: Consider the system $\Sigma=(A, B, C, D)$ and suppose the transfer function $C(z I-A)^{-1} B+D=0$. Then the following are equivalent.

i) The system $\Sigma=(A, B, C, D)$ is complete.

ii) $\operatorname{rank}\left[\begin{array}{cccc}0 & A^{n-1} B & \cdots & B \\ \vdots & & & \\ C A^{n-1} & & 0\end{array}\right]=n$.

iii) The reachable and the unobservable subspaces of $\Sigma$ are equal.

iv) The system $\Sigma=(A, B, C, D)$ is devoid of i.o.d. zeros.

Proof: Let $\mathcal{R}_{0}$ denote the reachable subspace and $\mathcal{N}_{0}$ the unobservable subspace of $\Sigma$, i.e.,

$$
\mathcal{R}_{0}=\sum_{i=0}^{n-1} A^{i} \operatorname{Im}(B), \quad \mathcal{N}_{0}=\bigcap_{i=0}^{n-1} \operatorname{Ker}\left(C A^{i}\right) .
$$

Since, by hypothesis, $D=0$ and $C A^{i} B=0$ for all nonnegative integers $i$, we have $\mathcal{R}_{0} \subset \mathcal{N}_{0}$. The system $\Sigma$ satisfies (ii) if and only if

$$
\operatorname{rank}\left[\begin{array}{lll}
A^{n-1} B & \cdots & B
\end{array}\right]+\operatorname{rank}\left[\begin{array}{lll}
C^{\prime} & \cdots & \left(C A^{n-1}\right)^{\prime}
\end{array}\right]^{\prime}=n,
$$

which is equivalent to $\operatorname{dim}\left[\mathcal{R}_{0}\right]=\operatorname{dim}\left[\mathcal{N}_{0}\right]$ or to $\mathcal{R}_{0}=\mathcal{N}_{0}$. Thus, (ii) is equivalent to (iii). To see the equivalence of (i) and (iii), by a suitable similarity transformation, we can transform $\Sigma$ into an equivalent system

$$
\left(\left[\begin{array}{cc}
A_{1} & 0 \\
A_{2} & A_{3}
\end{array}\right], \quad\left[\begin{array}{l}
B_{1} \\
B_{2}
\end{array}\right], \quad\left[\begin{array}{lll}
C_{1} & 0
\end{array}\right], \quad 0\right)
$$

such that $\left(C_{1}, A_{1}\right)$ is observable. By $\mathcal{R}_{0} \subset \mathcal{N}_{0}$, it is easily follows that $C_{1} A_{1}^{i} B_{1}=0$ for all nonnegative $i$, which implies by the observability of $\left(C_{1}, A_{1}\right)$ that $B_{1}=0$. Now, (iii) holds if and only if $\left(A_{3}, B_{2}\right)$ is reachable, which in turn is equivalent to

$$
\operatorname{rank}\left[\begin{array}{ccc}
z I-A_{1} & 0 & 0 \\
-A_{2} & z I-A_{3} & B_{2} \\
-C_{1} & 0 & 0
\end{array}\right]=n
$$

for all complex $z$. This is equivalent to (i) as completeness is invariant under system equivalence. This proves (i) $\Leftrightarrow$ (iii). Finally, we show (iii) $\Leftrightarrow$ (iv). The system has no i.o.d. zeros if and only if $\operatorname{dim}\left[\left(\mathcal{R}_{0}+\mathcal{N}_{0} / \mathcal{R}_{0}\right]=\operatorname{dim}\left[\mathcal{N}_{0} / \mathcal{R}_{0}\right]=0\right.$. The last equality holds if and only if (iii) holds.

Remark 1: The result of Lemma 1 can be extended to prove that if the transfer matrix is identically zero, then the incompleting zeros are precisely the i.o.d. zeros. We also state without proof that, in general, any $z \in C$ which is both a blocking zero [7] and an i.o.d. zero is also an incompleting zero of $\Sigma$. Thus, $\{$ blocking zeros $\} \cap$ \{i.o.d.zeros $\} \subseteq\{$ incompleting zeros $\}$. The reverse inclusion is true provided the transfer matrix is identically zero, but false in general as the following example shows. The system $\Sigma=$ $(A, B, C, D)$ with

$$
A=\left[\begin{array}{cc}
0 & 0.5 \\
0 & 0
\end{array}\right], B=\left[\begin{array}{l}
1 \\
0
\end{array}\right], C=\left[\begin{array}{ll}
0 & 1
\end{array}\right],
$$

$D=2$ has an incompleting zero at $z=0$, but has no blocking or i.o.d. zeros.

Consider now the time-invariant version of (1):

$$
\Sigma: \begin{array}{ll}
x(k+1) & =A x(k)+\sum_{j=1}^{\nu} B_{j} u_{j}(k), \\
y_{i}(k) & =C_{i} x(k)+\sum_{j=1}^{\nu} D_{i j} u_{j}(k), \quad i=1, \cdots, \nu .
\end{array}
$$

Let us define

$$
\begin{aligned}
& \Sigma_{i_{1} \cdots i_{\mu} j_{1} \cdots j_{\nu^{\prime}-\mu}}:=\left(A,\left[\begin{array}{llll}
B_{j 1} & B_{j 2} & \cdots & B_{j_{\nu-\mu}}
\end{array}\right],\left[\begin{array}{c}
C_{i_{1}} \\
C_{i_{2}} \\
\vdots \\
C_{i_{\mu}}
\end{array}\right],\right. \\
& \left.\left[\begin{array}{cccc}
D_{i_{1} j_{1}} & D_{i_{1} j_{2}} & \cdots & D_{i_{1} j_{\nu-\mu}} \\
D_{i_{2} j_{1}} & D_{i_{2} j_{2}} & \cdots & D_{i_{2} j_{\nu-\mu}} \\
\vdots & \vdots & & \vdots \\
D_{i_{\mu} j_{1}} & D_{i_{\mu} j_{2}} & \cdots & D_{i_{\mu} j_{\nu-\mu}}
\end{array}\right]\right)
\end{aligned}
$$

and let the associated transfer matrix be denoted by $Z_{i_{1} \cdots i_{\mu} j_{1} \cdots j_{\nu-\mu}}(z)$ for $\mu=1, \cdots, \nu$ and $i_{k}, j_{l} \in\{\mathbf{1}, \cdots, \nu\}$ such that $\left\{i_{1}, \cdots, i_{\mu}, j_{1}, \cdots, j_{\nu-\mu}\right\}=\{1, \cdots, \nu\}$. Such subsystems of $\Sigma$ are called the complementary subsystems in [3].

The TIDSP has the following well-known solution due to Wang and Davison [24], Anderson and Clements [1], and Davison and Chang [5].

Lemma 2: The TIDSP has a solution if and only if the system $\Sigma$ is stabilizable and detectable, i.e., for all $z \in \bar{D}$

$$
\begin{aligned}
& \operatorname{rank}\left[z I-A \quad B_{1} \quad B_{2} \quad \cdots \quad B_{\nu}\right]=n, \\
& \operatorname{rank}\left[z I-A^{\prime} \quad C_{1}^{\prime} \quad C_{2}^{\prime} \quad \cdots \quad C_{\nu}^{\prime}\right]^{\prime}=n,
\end{aligned}
$$

and all the $2^{\nu}-2$ complementary subsystems $\Sigma_{i_{1}, \cdots, i_{\mu}, j_{1}, \cdots, j_{\nu-\mu}}$ are weakly complete.

Any $z \in \bar{D}$ for which at least one of the rank conditions or the weak-completeness conditions above fails is an unstable decentralized fixed mode of the $\nu$-channel system. Thus, alternatively, TIDSP is 
solvable if and only if the system has no unstable decentralized fixed modes. If the solvability conditions of Lemma 2 hold, a solution can be obtained by following the procedures in any one of, e.g., [24], $[3],[22],[6],[15],[20]$.

III. Periodically Time-VARYing Systems AND LifTing

Suppose that a $p \times m$ system $\Sigma(k)$ given by

$$
\Sigma(k): \begin{array}{ll}
x(k+1) & =A(k) x(k)+B(k) u(k), \\
y(k) & =C(k) x(k)+D(k) u(k),
\end{array}
$$

is periodically time varying (PTV) with fundamental period $N$, i.e., $N$ is the smallest positive integer for which $A(k+N)=A(k)$, $B(k+N)=B(k), C(k+N)=C(k), D(k+N)=D(k)$, for all $k \geq 0$. Any multiple $M$ of $N$ with a positive integer is also a period, and we define the M-lifting of $\Sigma(k)$ to be the time invariant $p M \times m M$ system $\Sigma^{M}$ given by the equations

$$
\Sigma^{M}: \hat{x}(k+1)=F \hat{x}(k)+G \hat{u}(k),
$$

$k \geq 0$

where the matrices $F, G, H, J$ are given in terms of the state transition matrix $\Phi(k, l):=A(k-1) \cdots A(l)$ for $k>l$ and $\Phi(l, l)=I$ associated with $\Sigma(k)$ as follows:

$$
F:=\Phi(M, 0)
$$

$G:=\left[\begin{array}{lll}\Phi(M, 1) B(0) & \cdots & \Phi(M, M-1) B(M-2) \quad B(M-1)\end{array}\right]$,

$$
H:=\left[\begin{array}{c}
C(0) \\
C(1) \Phi(1,0) \\
\vdots \\
C(M-2) \Phi(M-2,0) \\
C(M-1) \Phi(M-1,0)
\end{array}\right]
$$

$$
J_{i j}= \begin{cases}0 & \text { if } i<j \\ D(i-1) & \text { if } i=j \\ C(i-1) \Phi(i-1, j) B(j-1) & \text { if } i>j\end{cases}
$$

with $J=\left[J_{i j}\right]$ for $i, j=1, \cdots, M$. Observe that $G$ is the $M$ step reachability matrix and $H$ is the $M$-step unobservability matrix associated with $\Sigma(k)$. It is easy to see that $\Sigma(k)$ is reachable in $n M$ steps if and only if $\Sigma^{M}$ is reachable. Similarly for observability. Moreover, if $\Sigma(k)=0$, i.e., if $\Sigma(k)$ is a zero input-output system or, equivalently, if $D(k)=0, C(k) \Phi(k, l) B(l-1)=0, \forall k \geq l \geq 0$, then the transfer matrix of $\Sigma^{M}$ is identically zero for all periods $M$. Conversely, if the latter is true for some period $M$, then $\Sigma(k)$ is a zero input-output system. The correspondence between a PTV system and its $M$-lifting is actually much stronger, and many system-theoretic questions regarding $\Sigma(k)$ can be settled via $\Sigma^{M}$. The following result of $[12]$ is the base for all subsequent discussions.

Lemma 3: Let $\Sigma(k)$ be a PTV system with period $M$, and let $\Sigma^{M}$ be its $M$-lifting. There exists a PTV controller internally u.a. stabilizing $\Sigma(k)$ if and only if there exists a TI controller internally stabilizing $\Sigma^{M}$.

Proof: The "only if" part of the claim is a direct consequence of [12, Lemma 2.7]. To see the "if" part, we note that a TI plant which admits an internally stabilizing TI controller can also be stabilized using a strictly proper controller. Thus, there exists a controller of transfer matrix $C(z)$, with $C(\infty)=0$, which internally stabilizes $\Sigma^{M}$. It now follows from the discussions in Section II and Lemma 2.7 of the aforementioned paper that $C(z)$ yields a PTV system which internally u.a. stabilizes $\Sigma(k)$.

Let us now consider a TI system

$$
\Sigma: \begin{array}{ll}
x(k+1) & =A x(k)+B u(k), \\
y(k) & =C x(k)+D u(k),
\end{array}
$$

and let $T(z):=C(z I-A)^{-1} B+D$ be its transfer matrix. Also, let $T_{i}:=C A^{i-1} B$ for $i \geq 1$ with $T_{0}:=D$. For this system, $N=1$ and any positive integer $\bar{M}$ is a period. Computing the $M$-lifting $\Sigma^{M}$ of this system via the procedure given above, we obtain (7), where the matrices $F, G, H, J$ take the special forms

$F=A^{M}, G=\left[\begin{array}{lll}A^{M-1} B & \cdots & B\end{array}\right]$,

$H=\left[\begin{array}{c}C \\ C A \\ \vdots \\ C A^{M-2} \\ C A^{M}\end{array}\right], J=\left[\begin{array}{ccccc}D & & & & \\ C B & D & & 0 & \\ \vdots & \vdots & \ddots & & \\ C A^{M-3} B & C A^{M-4} B & \cdots & D & \\ C A^{M-2} B & C A^{M-3} B & \cdots & C B & D\end{array}\right]$.

The relevance of periodic feedback to the incompleting zeros is now clarified by the next result, which is the central result of the paper.

Theorem 1: (i) Let $T(z)=0$. Then,

$$
\operatorname{rank}\left[\begin{array}{cc}
z I-A & B \\
-C & 0
\end{array}\right]=n \quad \forall z \in \bar{D}
$$

if (for some $M$ ) and only if (for all $M$ )

$$
\operatorname{rank}\left[\begin{array}{cc}
z I-F & G \\
-H & 0
\end{array}\right]=n \quad \forall z \in \bar{D} \text {. }
$$

(ii) Let $T(z) \neq 0$, and let $j$ be the smallest integer for which $T_{j} \neq 0$. Then,

$$
\operatorname{rank}\left[\begin{array}{cc}
z I-F & G \\
-H & J
\end{array}\right] \geq n \quad \forall z \in \bar{D}
$$

for some $M \leq j+1+M(j)$, where $M(j)$ is the greatest integer less than or equal to $n / \operatorname{rank}\left(T_{j}\right)$.

Proof: Consider an arbitrary system $\Sigma=(A, B, C, D)$ and its stable-antistable decomposition obtained by a similarity transformation, i.e., the system similar to $\Sigma$ obtained by letting $E$ be any nonsingular matrix such that

$$
C E=\left[\begin{array}{ll}
C_{s} & C_{a}
\end{array}\right], \quad E^{-1} A E=\left[\begin{array}{cc}
A_{s} & 0 \\
0 & A_{a}
\end{array}\right], \quad E^{-1} B=\left[\begin{array}{l}
B_{s} \\
B_{a}
\end{array}\right]
$$

where all the eigenvalues of $A_{s}$ in $D$ and all the eigenvalues of $A_{a}$ are in $\bar{D}$. It is easy to see that $(A, B, C, D)$ is weakly complete if and only if $\left(A_{a}, B_{a}, C_{a}, D\right)$ is complete. Also, $C(z I-$ $A)^{-1} B+D=T_{s}(z)+T_{a}(z)$, where $T_{s}(z):=C_{s}\left(z I-A_{s}\right)^{-1} B_{s}$, $T_{a}(z):=C_{a}\left(z I-A_{a}\right)^{-1} B_{a}+D$. Since the poles of $T_{s}(z)$ and $T_{a}(z)$ are disjoint, we also have that $C(z I-A)^{-1} B+D=0$ if and only if $T_{s}(z)=0$ and $T_{a}(z)=0$. We now prove (i). The hypothesis $T(z)=0$ implies that in the stable-antistable decomposition of $\Sigma$ as obtained above, $T_{s}(z)=0$ and $T_{a}(z)=0$, and that the condition (8) holds if and only if

$$
\operatorname{rank}\left[\begin{array}{cc}
z I-A_{a} & B_{a} \\
-C_{a} & 0
\end{array}\right]=n_{a} \quad \forall z \in C
$$

where $n_{a}, n_{s}$ respectively denote the dimensions of $A_{a}, A_{s}$. Applying Lemma 1, (8) holds if and only if

$$
\operatorname{rank}\left[\begin{array}{cccc}
0 & A_{a}^{n_{a}-1} B_{a} & \cdots & B_{a} \\
C_{a} & & & \\
\vdots & & 0
\end{array}\right]=n_{a} \quad \forall z \in C
$$

where $n_{a}:=\operatorname{size} A_{a}$. We now show that (11) holds if and only if (9) holds. Let us define

$$
\begin{gathered}
F_{i}:=A_{i}^{M}, G_{i}:=\left[\begin{array}{llll}
A_{i}^{m-1} B_{i} & \cdots & B_{i}
\end{array}\right], \\
H_{i}:=\left[\begin{array}{lll}
C_{i}^{\prime} & \cdots & \left(C_{i} A_{i}^{M-1}\right)^{\prime}
\end{array}\right]^{\prime}
\end{gathered}
$$


for $i=s, a$. By a straightforward computation, it is easily checked that $T_{s}^{M}(z)=H_{s}\left(z I-F_{s}\right)^{-1} G_{s}$ and $T_{a}^{M}(z)=$ $H_{a}\left(z I-F_{a}\right)^{-1} G_{a}+J$ is a stable-antistable decomposition of the $M$-lifting $\Sigma^{M}$. Moreover, again by the hypothesis, the transfer matrix of the lifted system is identically zero, implying that $T_{a}^{M}(z)=0$. By the discussion at the beginning of the proof, we have that (9) holds if and only if $\left(F_{a}, G_{a}, H_{a}\right)$ is complete. By Lemma 1 , this holds if and only if

$$
\operatorname{rank}\left[\begin{array}{cccc}
0 & F_{a}^{n_{a}-1} G_{a} & \cdots & G_{a} \\
H_{a} & & & \\
\vdots & & 0
\end{array}\right]=n_{a} \quad \forall z \in C .
$$

By the Cayley-Hamilton theorem, it now follows that (11) and (12) are equivalent statements. This completes the proof of (i). We now prove (ii). By the hypothesis, the matrix $J$ has the form $J=\left[J_{k l}\right]$ for $k, l=1, \cdots, M$, where $J_{k l}=0$ if $k-l<j$ and $J_{k l}=T_{l}$ if $k-l \geq j$, i.e., all the entries above the $j$ th block subdiagonal are zero and the block entries at the $j$ th subdiagonal are all $T_{j}$. Hence, $\operatorname{rank}(J)=(M-j) \operatorname{rank}(T j)$. If we choose $M=j+1+M(j)$, then we have $\operatorname{rank}(J) \geq n$, which assures that $(10)$ holds

Remark 2: a) The interpretation of Theorem 1 is that: (i) the incompleting zeros are invariant under lifting for systems of zero transfer matrix, and (ii) the incompleting zeros can be eliminated via lifting for systems of nonzero transfer matrix. The ability of periodic control to eliminate blocking zeros and to relocate some other zeros has been noted before; see [12] and [13]. Theorem 1 shows the role of periodic feedback in relation to incompleting zeros and, in view of Remark 1 above, we conclude that there is more to the power of periodic control than what has been noticed in the literature so far. b) Using Lemma 1 and the stable-antistable decomposition in the above proof, it is straightforward to prove that if $T(z)=0$, then the system $\sum$ is weakly complete if and only if it is devoid if i.o.d. zeros in $\overline{\boldsymbol{D}}$ (or unstable i.o.d. zeros). In view of this and Remark 1, Theorem 1(i) reads as: The unstable i.o.d. zeros of a system with identically zero input-output map are invariant under lifting.

The fact that stabilizability and detectability are preserved under lifting can be recovered from Theorem 1 (i) as follows. Since $(A, B, C, D)$ is stabilizable if and only if $(A, B, 0,0)$ is stabilizable, statement (i) of Theorem 1 yields, in particular, that a system is stabilizable just in case its M-lifting is stabilizable; similarly for detectability.

\section{PTV CONTROLlers for TI Plants}

Let us again consider the TI $\nu$-channel plant (6) and its subsystems $\left(A, B_{j}, C_{i}, D_{i j}\right)$. Let their $M$-liftings be denoted by $\left(F, G_{j}, H_{i}, J_{i j}\right)$ for $i, j=1, \cdots, \nu$. The $p M \times m M$ system, where $p:=\sum_{i=1}^{\nu} p_{i}$ and $m:=\sum_{i=1}^{\nu} m_{i}$, given by the equations

$$
\begin{aligned}
& \Sigma^{M, \pi}: \begin{array}{l}
\hat{x}(k+1)=F \hat{x}(k)+\sum_{j=1}^{\nu} G_{j} \hat{u}_{j}(k), \\
\hat{y}_{i}(k)=H_{i} \hat{x}(k)+\sum_{j=1}^{\nu} J_{i j} \hat{u}_{j}(k),
\end{array} \\
& i=1, \cdots, \nu
\end{aligned}
$$

is an $M$-lifting of (6) modulo a reordering at input and output channels. The superscript $\pi$ is included to emphasize the fact that $\Sigma^{M, \pi}$ is an $M$-lifting of the system (6) followed by a permutation at the input and output channels.

Proposition 1: There exists a PTV decentralized controller of period $M$ solving TVDSP for the system (6) if and only if TIDSP is solvable for the system (13).

Proof: We prove the claim for the case $\nu=2, M=2$. The generalization of the argument to an arbitrary number of channels and to any $M$ is straightforward. Let

$$
\begin{aligned}
\left(\left[\begin{array}{cc}
\bar{A}_{1}(k) & 0 \\
0 & \bar{A}_{2}(k)
\end{array}\right],\right. & {\left[\begin{array}{cc}
\bar{B}_{1}(k) & 0 \\
0 & \bar{B}_{2}(k)
\end{array}\right], } \\
& {\left.\left[\begin{array}{cc}
C_{1}(k) & 0 \\
0 & \bar{C}_{2}(k)
\end{array}\right],\left[\begin{array}{cc}
\bar{D}_{1}(k) & 0 \\
0 & \bar{D}_{2}(k)
\end{array}\right]\right) }
\end{aligned}
$$

be an $M$-periodic solution to TVDSP for (6) with $\nu=2$. By Lemma 3 , the $M$-lifting $(\bar{F}, \bar{G}, \bar{H}, \bar{J})$ of this controller, where

$$
\begin{aligned}
& \bar{F}:=\left[\begin{array}{cc}
\bar{\Phi}_{1}(2,0) & 0 \\
0 & \bar{\Phi}_{2}(2,0)
\end{array}\right], \\
& \bar{G}:=\left[\begin{array}{cccc}
\bar{\Phi}_{1}(2,1) \bar{B}_{1}(0) & 0 & \bar{B}_{1}(1) & 0 \\
0 & \bar{\Phi}_{2}(2,1) \bar{B}_{2}(0) & 0 & \bar{B}_{2}(1)
\end{array}\right], \\
& \bar{H}:=\left[\begin{array}{cc}
\bar{C}_{1}(0) & 0 \\
0 & \bar{C}_{2}(0) \\
\bar{C}_{1}(1) \bar{\Phi}_{1}(1,0) & 0 \\
0 & \bar{C}_{2}(1) \bar{\Phi}_{2}(1,0)
\end{array}\right] \text {, } \\
& \bar{J}:=\left[\begin{array}{cccc}
\bar{D}_{1}(0) & 0 & 0 & 0 \\
0 & \bar{D}_{2}(0) & 0 & 0 \\
\bar{C}_{1}(1) \bar{B}_{1}(0) & 0 & \bar{D}_{1}(1) & 0 \\
0 & \bar{C}_{2}(1) \bar{B}_{2}(0) & 0 & \bar{D}_{2}(1)
\end{array}\right],
\end{aligned}
$$

and $\bar{\Phi}_{i}(\cdot, \cdot)$ is the transition matrix associated with $\bar{A}_{i}(\cdot)$ for $i=1,2$, internally u.a. stabilizes the $M$-lifting of (6) which is $(F, G, H, J)$, where

$$
\begin{aligned}
& F:=A^{2}, G:=\left[\begin{array}{llll}
A B_{1} & A B_{2} & B_{1} & B_{2}
\end{array}\right], \\
& H:=\left[\begin{array}{c}
C_{1} \\
C_{2} \\
C_{1} A \\
C_{2} A
\end{array}\right], J:=\left[\begin{array}{cccc}
D_{11} & D_{12} & 0 & 0 \\
D_{21} & D_{22} & 0 & 0 \\
C_{1} B_{1} & C_{1} B_{2} & D_{11} & D_{12} \\
C_{2} B_{1} & C_{2} B_{2} & D_{21} & D_{22}
\end{array}\right] .
\end{aligned}
$$

It follows by suitable permutations at the input and output channels that the decentralized TI controller $\left(\bar{F}, b \operatorname{diag}\left\{\bar{G}_{1}, \bar{G}_{2}\right\}\right.$, $\left.b \operatorname{diag}\left\{\bar{H}_{1}, \bar{H}_{2}\right\}, b \operatorname{diag}\left\{\bar{J}_{1}, \bar{J}_{2}\right\}\right)$, where $\bar{F}$ is as above and

$$
\begin{aligned}
\bar{G}_{i}: & =\left[\begin{array}{ll}
\bar{\Phi}_{i}(2,1) \bar{B}_{i}(0) & \bar{B}_{i}(1)
\end{array}\right], \\
\bar{H}_{i}: & =\left[\begin{array}{cc}
\bar{C}_{i}(0) \\
\bar{C}_{i}(1) \bar{\Phi}_{i}(1,0)
\end{array}\right], \\
J_{i} & :=\left[\begin{array}{cc}
\bar{D}_{i}(0) & 0 \\
\bar{C}_{i}(1) \bar{B}_{i}(0) & \bar{D}_{i}(1)
\end{array}\right],
\end{aligned}
$$

internally stabilizes the plant

$$
\left(F,\left[\begin{array}{ll}
F_{1} & F_{2}
\end{array}\right],\left[\begin{array}{l}
H_{1} \\
H_{2}
\end{array}\right],\left[\begin{array}{ll}
J_{11} & J_{12} \\
J_{21} & J_{22}
\end{array}\right]\right),
$$

proving one part of our claim. Conversely, let there exist a TI decentralized stabilizing controller for (14). If we denote the transfer matrix of (14) by $T(z)$, then there also exists a TI decentralized controller for the plant of transfer matrix $z^{-1} T(z)$, say $C(z)$, since the addition of a pole at the origin would not affect the weakcompleteness conditions of Lemma 2 . It follows that the strictly proper decentralized controller $z^{-1} C(z)$ internally stabilizes $T(z)$. Suitable permutations at the input and output channels now yield a strictly proper internally stabilizing controller for $(F, G, H, J)$. By Lemma 3 and the procedure in the construction part of its proof, this yields a 2-periodic controller that has decentralized structure and that internally stabilizes (6).

Combining the results of Theorem 1 and Proposition 1, we obtain the main result of this section.

Theorem 2: There exists a PTV decentralized controller that internally stabilizes the $\nu$-channel TI system of (6) if and only if the composite system is stabilizable and detectable (by a centralized controller), and each complementary subsystem of $\Sigma$ satisfies

$Z_{i_{1}, \cdots, i_{\mu}, j_{1}, \cdots, j_{\nu-\mu}}=0 \Rightarrow \Sigma_{i_{1}, \cdots, i_{\mu}, j_{1}, \cdots, j_{\nu-\mu}}$ is weakly complete 
Proof: By Proposition 1, there exists a periodic solution to TVDSP for the periodic plant (6) if and only if there exists $M$ and a TI decentralized controller for the system $\Sigma^{M, \pi}$ of (13). By Lemma 2 , such a TI decentralized controller exists if and only if there exists $M$ such that the following four conditions are satisfied by (13).

C1) The composition system $\Sigma^{M, \pi}$ is stabilizable (by a centralized controller).

C2) The composite system $\Sigma^{M, \pi}$ is detectable (by a centralized controller).

C3) If $Z_{i_{1}, \cdots, i_{\mu}, j_{1}, \cdots, j_{\nu-\mu}}^{M, \pi}(z)=0$, then $\Sigma_{i_{1}, \cdots, i_{\mu}, j_{1}, \cdots, j_{\nu-\mu}}^{M, \pi}$ is weakly complete.

C4) If $Z_{i_{1}, \cdots, i_{\mu}, j_{1}, \cdots, j_{\nu-\mu}}^{M,}(z) \neq 0$, then $\Sigma_{i_{1}, \cdots, i_{\mu}, j_{1}, \cdots, j_{\nu-\mu}}^{M, \pi}$ is weakly complete.

The last two conditions are to be satisfied for all complementary subsystems.

The stabilizability and detectability conditions $\mathrm{C} 1$ ) and $\mathrm{C} 2$ ) on the composite system hold if and only if the original system (6) is stabilizable and detectable. This is by the last two statements of Section III and by the fact that stabilizability and detectability are preserved under input or output permutations. The system matrix associated with $\Sigma_{i_{1}, \cdots, i_{\mu}, j_{1}, \cdots, j_{\nu-\mu}}^{M, \pi}$ is given by

$$
\left[\begin{array}{cccc}
z I-F & G_{j_{1}} & \cdots & G_{j_{\nu-\mu}} \\
-H_{i_{1}} & J_{i_{1} j_{1}} & \cdots & J_{i_{1} j_{\nu-\mu}} \\
\vdots & \vdots & & \vdots \\
-H_{i_{\mu}} & J_{i_{\mu} j_{1}} & \cdots & J_{i_{\mu} j_{\nu-\mu}}
\end{array}\right]
$$

or, substituting the expressions for $F, G_{j}, H_{i}, J_{i j}$ in terms of $A$, $B_{j}, C_{i}, D_{i j}$ and performing suitable row and column permutations, as shown by the matrix at the bottom of the page.

We now recognize this system matrix as the system matrix associated with the $M$-lifting of the complementary subsystem $\Sigma_{i_{1}, \cdots, i_{\mu}, j_{1}, \cdots, j_{\nu-\mu}}$ of the original system (6): Applying Theorem 1(i), we have that $\mathrm{C} 3$ ) holds for some (and hence all) $M$ if and only if $\Sigma_{i_{1}, \cdots, i_{\mu}, j_{1}, \cdots, j_{\nu-\mu}}$ is weakly complete. On the other hand, by Theorem 1(ii), there exists an integer $M_{i_{1}, \cdots, i_{\mu}, j_{1}, \cdots, j_{\nu-\mu}}$ for which the condition C4) is satisfied. If we let $M:=\max \left\{M_{i_{1}}, \cdots, i_{\mu}, j_{1}, \cdots, j_{\nu-\mu}\right\}$ as the index assumes all possible values, we have that $\mathrm{C} 4$ ) is satisfied for all complementary subsystems. Consequently, there exists a TI decentralized controller internally stabilizing (13) if and only if the conditions of Theorem 2 hold. By Proposition 1, the same conditions constitute necessary and sufficient conditions for the existence of an $M$-periodic decentralized controller internally stabilizing the original TI plant (6)

Remark 3: Note that if the conditions of Theorem 2 hold, then there is a PTV controller of period at most $M=\max \left\{M_{i_{1}, \cdots, i_{\mu}, j_{1}, \cdots, j_{\nu-\mu}}\right\}$ where the maximum is taken with respect to all indexes, yielding a complementary subsystem of (6), and each $M_{i_{1}, \cdots, i_{\mu}, j_{1}, \cdots, j_{\nu-\mu}}$ is computed by the upper bound given in Theorem 1(ii).
Remark 4: Theorem 2 is a generalization of the main result of [2] in the discrete-time case. Note that if the system (6) is canonical and strongly connected, then it has no i.d. zeros, no o.d. zeros, and all complementary subsystems are nonzero. In this case, a periodic decentralized controller exists.

\section{PTV CONTROLLERS FOR PTV PLANTS}

Let us now consider the $\nu$-channel TV system $\Sigma(k)$ of (1) and assume that $\Sigma(k)$ is periodic with fundamental period $N$. We are now interested in the existence of $\nu$ local controllers $\bar{\Sigma}_{i}(k), i=1, \cdots, \nu$ as in (2) which are periodically time varying and which solve TVDSP for (1).

Let $\left(A, B_{j}, C_{i}, D_{i j}\right)$ be the $N$-lifting of the subsystem $(A(k)$, $\left.B_{j}(k), C_{i}(k), D_{i j}(k)\right)$ for $i, j=1, \cdots, \nu$ and consider the $\nu$ channel time-invariant system obeying

$$
\begin{aligned}
& \Sigma^{N, \pi}: \begin{array}{lll}
\hat{x}(k+1) & =A \hat{x}(k)+\sum_{j=1}^{\nu} & B_{j} \hat{u}_{j}(k), \\
\hat{y}_{i}(k) & = & C \\
& & \hat{x}(k)+\sum_{j=1}^{\nu} D_{i j} \hat{u}_{j}(k),
\end{array} \\
& i=1, \cdots, \nu
\end{aligned}
$$

Our first result transforms the problem to determining a PTI controller for the TI plant $\Sigma^{N, \pi}$, which is the $N$-lifting of (1) followed by suitable permutations at the input and output channels.

Proposition 2: There exist PTV controllers (2) solving TVDSP for the plant (1) if and only if there exist PTV controllers of the type

$$
\tilde{\Sigma}_{i}(k): \begin{array}{lll}
\tilde{x}_{i}(k+1) & =\tilde{A}_{i}(k) \tilde{x}_{i}(k)+\tilde{B}_{i}(k) \hat{y}_{i}(k), \\
\hat{u}_{i}(k) & =\tilde{C}_{i}(k) \tilde{x}_{i}(k)+\tilde{D}_{i}(k) \hat{y}_{i}(k), \quad i=1, \cdots, \nu
\end{array}
$$

internally stabilizing the TI plant $\Sigma^{N, \pi}$ of (15).

Proof: We only give an outline of the proof since a rigorous proof is notationally complex mainly due to the permutations involved to ensure the decentralized structure of controllers. Let $\bar{\Sigma}(k)$ be a $K$ periodic controller solving TVDSP for $\Sigma(k)$. Let $M$ be the least common multiple of the integers $N$ and $K$. Then, both systems are $M$-periodic, and by Lemma $3, \bar{\Sigma}^{M, \pi}$ is a solution to TIDSP for $\Sigma^{M, \pi}$. Note that $\Sigma^{M, \pi}$ is an $M / N$-lifting of $\Sigma^{N, \pi}$ followed by a permutation at the input and output channels. By Proposition 1 , it follows that there exists a PTV solution to TVDSP for $\Sigma^{N, \pi}$. Conversely, let $\Sigma^{N, \pi}$ admit a PTV solution of period $K$ to TVDSP. Let $M$ be as before, and note that $M / N$-lifting of $\Sigma^{N, \pi}$ is an $M$ lifting of $\Sigma(k)$ followed by some suitable permutations at the input and output channels. By Proposition 1, this $M$-lifting of $\Sigma(k)$ admits a TI-decentralized solution to TIDSP. Again, by Proposition 1, $\Sigma(k)$ has a PTV decentralized solution to TVDSP.

We can now use Theorem 2 to get the following result.

Theorem 3: There exists a PTV decentralized controller internally stabilizing the PTV system $\Sigma$ of (1) if and only if $\Sigma$ is stabilizable and detectable, and for each complementary subsystem of $\Sigma$,

$f\left(\Sigma_{i_{1}, \cdots, i_{\mu}, j_{1}, \cdots, j_{\nu-\mu}}\right)=0 \Rightarrow \Sigma_{i_{1}, \cdots, i_{\mu}, j_{1}, \cdots, j_{\nu-\mu}}^{N}$ is weakly complete

$$
\left[\begin{array}{cccccccc}
z I-A^{M} & A^{M-1} B_{j_{1}} & \cdots & A^{M-1} B_{j_{1}} & \cdots & B_{j_{\nu-\mu}} & \cdots & B_{j_{\nu-\mu}} \\
-C_{i_{1}} & D_{i_{1} j_{1}} & \cdots & D_{i_{1} j_{\nu-\mu}} & & & & \\
\vdots & \vdots & & \vdots & & & 0 & \\
-C_{i_{\mu}} & D_{i_{\mu} j_{1}} & \cdots & D_{i_{\mu} j_{\nu-\mu}} & & & & \\
\vdots & & \vdots & & \ddots & & & \\
-C_{i_{1}} A^{M-1} & C_{i_{1}} A^{M-2} B_{j_{1}} & \cdots & C_{i_{1}} A^{M-2} B_{j_{\nu-\mu}} & & D_{i_{1} j_{1}} & \cdots & D_{i_{1} j_{\nu-\mu}} \\
\vdots & \vdots & & \vdots & \cdots & \vdots & & \vdots \\
-C_{i_{\mu}} A^{M-1} & C_{i_{\mu}} A^{M-2} B_{j_{1}} & \cdots & C_{i_{\mu}} A^{M-2} B_{j_{\nu-\mu}} & & D_{i_{\mu} j_{1}} & \cdots & D_{i_{\mu} j_{\nu-\mu}}
\end{array}\right] .
$$


where $f\left(\Sigma_{i_{1}, \cdots, i_{\mu}, j_{1}, \cdots, j_{\nu-\mu}}\right)$ is the input/output map of the complementary subsystem $\Sigma_{i_{1}, \cdots, i_{\mu}, j_{1}, \cdots, j_{\nu-\mu}}$ of $\Sigma$ and $\Sigma_{i_{1}, \cdots, i_{\mu}, j_{1}, \cdots, j_{\nu-\mu}}^{N}$ is its $N$-lifting.

Proof: By Proposition 2, (1) is stabilizable by a PTV decentralized controller if and only if $\Sigma^{N, \pi}$ is. By Theorem 2, the latter is possible if and only if $\Sigma^{N, \pi}$ is stabilizable and detectable and each of its complementary subsystem satisfies

$$
Z_{i_{1}, \cdots, i_{\mu}, j_{1}, \cdots, j_{\nu-\mu}}^{N, \pi}=0 \Rightarrow \Sigma_{i_{1}, \cdots, i_{\mu}, j_{1}, \cdots, j_{\nu-\mu}}^{N, \pi} \text { is weakly complete. }
$$

Now, $\Sigma^{N, \pi}$ is stabilizable and detectable if and only if $\Sigma^{N}$ is which, by Lemma 6 , is the case if and only if $\Sigma(k)$ is stabilizable and detectable. On the other hand, the implication (17) is satisfied for each complementary subsystem if and only if each complementary subsystem of $\Sigma^{N}$ satisfies the same implication (dropping the superscript " $\pi$ "). Finally, note that $Z_{i_{1}, \cdots, i_{\mu}, i_{1}, \cdots, j_{\nu-\mu}}^{N}=0 \Leftrightarrow$ $f\left(\Sigma_{i_{1}}, \cdots, i_{\mu}, j_{1}, \cdots, j_{\nu-\mu}\right)=0$, which proves the claim.

Note that if $\Sigma$ is reachable in $n N$-steps and observable in $n N$ steps, then $\Sigma^{N}$ is reachable and observable so that the first two conditions, stabilizability and detectability of $\Sigma(k)$, are satisfied. If, in addition, $\Sigma(k)$ is strongly connected, i.e., none of the complementary subsystems is a zero input-output system, then the third condition is trivially satisfied. Consequently, a strongly connected and canonical PTV system can always be internally stabilized by a PTV decentralized controller.

In conclusion, we have used the lifting technique for the synthesis of periodic controllers for decentralized stabilization. Our technique has given a complete solution to this problem.

\section{REFERENCES}

[1] B. D. O. Anderson and D. J. Clements, "Algebraic characterization of fixed modes in decentralized control," Automatica, vol. 17, pp. 703-712, 1981.

[2] B. D. O. Anderson and J. B. Moore, "Time-varying feedback laws for decentralized control," IEEE Trans. Automat. Contr., vol. AC-26, no. 5 , pp. 1133-1139, 1981.

[3] J. P. Corfmat and A. S. Morse, "Decentralized control of linear multivariable systems," Automatica, vol. 8, pp. 479-485, 1976

[4] J. H. Davis, "Stability conditions derived from spectral theory: Discrete systems with periodic feedback," SIAM J. Contr., vol. 10, pp. 1-13.

[5] E. J. Davison and T. N. Chang, "Decentralized stabilization and pole assignment for general proper systems," IEEE Trans. Automat. Contr., vol. 35 , pp. 652-664, 1990.

[6] C. A. Desoer and N. Gündeş, Algebraic Theory of Linear Feedback Systems with Full and Decentralized Controllers. Berlin, SpringerVerlag, 1990.

[7] P. G. Ferreira and S. P. Bhattacharyya, "On blocking zeros," IEEE Trans. Automat. Contr., pp. 258-259, Apr. 1977.

[8] G. A. Hewer, "Periodicity, detectability and the matrix Riccati equation," SIAM J. Contr. Optimiz., vol. 13, pp. 1235-1251, 1975.

[9] Y. Juan and P. T. Kabamba, "Simultaneous pole assignment in linear periodic systems using constrained structure feedback," IEEE Trans. Automat. Contr., vol. 34, pp. 168-173, 1989.

[10] E. I. Jury and F. J. Mullin, "The analysis of sampled-data control system with a periodically time-varying sampling rate," IRE Trans. Automat. Contr., vol. AC-24, pp. 15-21, 1959.

[11] H. Kano and T. Nishimura, "Periodic solutions of matrix Riccati equation with detectability and stabilizability," Int. J. Contr., vol. 29, pp. 471-487, 1979.

[12] P. P. Khargonekar, K. Poolla, and A. Tannenbaum, "Robust control of linear time-invariant plants using periodic compensation," IEEE Trans. Automat. Contr., vol. AC-30, no. 11, pp. 1088-1096, 1985.

[13] S. Lee, S. M. Meerkov, and T. Runolfsson, "Linear periodic feedback: Zeros placement capabilities," in Proc. 25th Conf. Decision Contr. Athens, Greece, Dec. 1986, pp. 2202-2207.
[14] R. A. Meyer and C. S. Burrus, "A unified analysis of multirate and periodically time-varying digital filters," IEEE Trans. Circuits Syst., vol. CAS-22, pp. 162-168, 1975.

[15] A. B. Özgüler, "Decentralized control: A stable proper fractional approach," IEEE Trans. Automat. Contr., vol. 35, no. 10, pp. 1109-1117, 1990.

[16] H. H. Rosenbrock, State Space and Multivariable Theory. New York: Wiley-Interscience, 1970.

[17] M. A. Rotea and P. P. Khargonekar, "Stabilizability of linear timevarying and uncertain linear system," IEEE Trans. Automat. Contr., vol. 33 , pp. 884-887, 1988.

[18] B. Sz.-Nagy and C. Foias, Harmonic Analysis of Operators on Hilbert Space. New York: American Elsevier, 1970.

[19] L. Travé, A. M. Tarras, and A. Titli, "An application of vibrational control to cancel unstable decentralized fixed modes," in Proc. IFAC World Congr. Oxford: Pergamon, 1985.

[20] K. Ünyelioğlu and A. B. Özgüler, "Decentralized stabilization: Characterization of all solutions and genericity aspects," Int. J. Contr., vol. 55, no. 6, pp. 1381-1403, 1992.

[21] K. Unyelioğlu, A. B. Özgüler, and P. P. Khargonekar, "Decentralized simultaneous stabilization and reliable control using periodic feedback," Syst. Contr. Lett., vol. 18, pp. 23-31, 1992.

[22] M. Vidyasagar and N. Viswanadham, "Algebraic characterization of decentralized fixed modes and pole assignment," Rep. 82-06, Univ. Waterloo, Ont., Canada, N2L 3G1, 1982.

[23] S. H. Wang, "Stabilization of decentralized control systems via timevarying controllers," IEEE Trans. Automat. Contr., vol. AC-27, pp. 741-744, June 1982.

[24] S. H. Wang and E. J. Davison, "On the stabilization of decentralized control systems," IEEE Trans. Automat. Contr., vol. AC-18, pp. 473-478, 1973.

[25] J. L. Willems, "Time-varying feedback for the stabilization of fixed modes in decentralized control systems," Automatica, vol. 25, no. 1, pp. 127-131, 1989.

\section{A Generalization of the Positive Real Lemma}

Rudolf Scherer and Werner Wendler

Abstract- The positive real lemma (also called the KalmanYacubovich-Popov lemma) characterizes the positive realness of the transfer function matrix of a linear dynamic system by algebraic conditions. In the case of a pole-zero cancellation appearing in the transfer function matrix, or in other words, missing the assumptions of controllability and observability, there exist generalized versions, which are discussed and proven applying the Kalman canonical decomposition.

\section{INTRODUCTION}

Consider the linear time-invariant system $\{F, G, H\}$ specified by

$$
\dot{x}=F x+G u, \quad y=H x
$$

where $x$ is an $n$-dimensional state vector, $u$ is a $p$-dimensional input vector, $y$ is a $p$-dimensional output vector, and $F, G$, and $H$ are real constant $n \times n, n \times p$, and $p \times n$ matrices, respectively. The transfer function matrix is the $p \times p$ rational matrix

$$
Z(s)=H(s I-F)^{-1} G .
$$

Manuscript received May 18, 1992; revised December 22, 1992 and May $14,1993$.

The authors are with the Institut für Praktische Mathematik, Universität Karlsruhe, D-76128 Karlsruhe, Germany.

IEEE Log Number 9216454 